\title{
Temporal Variations of Skin Pigmentation in C57BI/6 Mice Affect Optical Bioluminescence Quantitation
}

\author{
Allison Curtis, ${ }^{1}$ Katherine Calabro, ${ }^{3}$ Jean-Rene Galarneau, ${ }^{2}$ Irving J. Bigio, ${ }^{3,4}$ \\ Thomas Krucker ${ }^{1}$ \\ ${ }^{1}$ Global Imaging Group, Novartis Institutes for BioMedical Research, 250 Massachusetts Avenue, Cambridge, MA, 02139, USA \\ ${ }^{2}$ Translational Sciences, Novartis Institutes for BioMedical Research, Inc., Cambridge, MA, USA \\ ${ }^{3}$ Department of Biomedical Engineering, Boston University, Boston, MA, USA \\ ${ }^{4}$ Department of Electrical and Computer Engineering, Boston University, Boston, MA, USA
}

\begin{abstract}
Purpose: Depilation-induced skin pigmentation in C57BI/6 mice is a known occurrence, and presents a unique problem for quantitative optical imaging of small animals, especially for bioluminescence. The work reported here quantitatively investigated the optical attenuation of bioluminescent light due to melanin pigmentation in the skin of transgenic C57BI/6 mice, modified such that luciferase expression is under the transcription control of a physiologically and pharmacologically inducible gene.

Procedure: Both in vivo and ex vivo experiments were performed to track bioluminescence signal attenuation through different stages of the mouse hair growth cycle. Simultaneous reflectance measurements were collected in vivo to estimate melanin levels.

Results: Biological variability of skin pigmentation was found to dramatically affect collected bioluminescent signal emerging through the skin of the mice. When compared to signal through skin with no pigmentation, the signal through highly pigmented skin was attenuated an average of $90 \%$. Positive correlation was found between reflectance measurements and bioluminescence signal loss. A correction scheme is proposed based on this correlation, but signal variation due to non-melanin scattering and absorption sources introduce significant errors. Advanced spectral reflectance analysis will be necessary to develop a more reliable correction method in the future.

Conclusion: Skin pigmentation is a significant variable in bioluminescent imaging, and should be considered in experimental design and implementation for longitudinal studies, and especially when sensitivity to small signal changes, or differences among animals, is required.
\end{abstract}

Key Words: Depilation, C57BI/6 Mice, Melanin, Reflectance spectroscopy, Optical imaging

\section{Introduction}

Tn drug discovery and development, quantitative molecular imaging of small animals, such as bioluminescence

Correspondence to: Thomas Krucker; e-mail: thomas.krucker@novartis. com imaging (BLI) and fluorescence imaging (FLI), is used to non-invasively measure specific cellular and molecular events in investigating a variety of disease processes [1-9]. Bioluminescent light is produced through the oxidation of luciferin in the presence of oxygen, adenosine triphosphate, magnesium, and luciferase [1,8]. In creating transgenic reporter animals, to test whether a drug in fact activated a gene, luciferase expression can be under the transcription 
control of a gene of interest such that in vivo luciferase production reports specific gene activation that is quantifiable. $\mathrm{C} 57 \mathrm{Bl} / 6$ mice are the preferred background for genetically engineered transgenic mice modeling disease, but due to the dark color of their hair, hair removal by shaving and/or chemical depilation prior to imaging is required to allow for maximum signal collection [2]. However, hair removal disrupts the normal hair growth cycle, leading to variations in skin pigmentation.

The hair growth cycle consists of three phases: a resting telogen phase, where $\mathrm{C} 57 \mathrm{Bl} / 6$ skin is a pale pink color, an active hair growth anagen phase, where the skin becomes dark gray or black, and finally, a catagen phase where hair growth stops, and the skin transitions back to the telogen phase, returning to a pale pink color [10]. Depilation triggers the transition from the resting telogen phase to the active anagen phase, and thus a transition from light skin to dark skin. The dark pigmentation may result from the collection of melanin in the hair follicles, in preparation for new hair growth during the anagen phase [10].

Because bioluminescent signal travels through the tissue and skin of an animal before being collected, it is subject to optical scattering and absorption interactions along its path. These interactions alter the intensity and distribution of signal emerging from the animal which is ultimately imaged by the detector. The presence of melanin not only makes the skin appear darker, but it is suspected to be a significant source of bioluminescence signal attenuation due to its high absorption coefficient [11]. More importantly, variability in melanin concentration, either among animals or over time, leads to variations in the degree of signal attenuation. Such variability can result in misleading interpretations of the actual bioluminescent signal, introducing significant experimental error in in vivo studies. This is particularly problematic for long-term in vivo studies, where it is necessary to image the same animal multiple times over the course of several weeks, and for which depilation is required every 12 weeks. During an 8-week bioluminescent imaging study involving $\mathrm{C} 57 \mathrm{Bl} / 6$ transgenic mice, pigmentation variability was noted, with a significant increase in pigmentation during week 3 of the study. In such a case, the data collected at different time points are not comparable because the signals were subject to different amounts of attenuation. This limitation in BLI has been discussed [2, 3, 7, 12], but never fully investigated. Hemoglobin is another strong absorber, especially in the wavelength region between 500 and $600 \mathrm{~nm}$, which is known to attenuate bioluminescent signal [12]. However, assuming similar blood perfusion over time, this should not affect the measure of signal change due to melanin absorption.

FLI can mitigate the problem of signal absorption due to the fact that the absorption coefficient of melanin decreases with wavelength. Thus, light at shorter wavelengths (i.e., in the visible spectrum) is more sensitive to attenuation than light at near-infrared wavelengths. Fluorescence imaging can use near-infrared dyes that have emission spectra at longer wavelengths, making them less sensitive to melanin absorption [13]. BLI, however, does not have this advantage. The emission peak of firefly luciferase is approximately $560 \mathrm{~nm}$, subjecting the bioluminescent signal to significant scattering and absorption effects from the tissue it interacts with [1, 12]. Previous work has been done to reduce light scattering in tissue during BLI by applying hyperosmotic agents to the skin, which reduce the scattering coefficient, thereby improving spatial resolution [14]. However, this approach does not address the more significant effects of melanin absorption, and was found to dramatically decrease collected bioluminescent signal. Although not investigated here, another approach to avoid the onset of depilation-induced skin pigmentation is through breeding the $\mathrm{C} 57 \mathrm{Bl} / 6$ mice with $\mathrm{C} 57$ albino mice, producing offspring that no longer have dark brown fur [15]. In this case, depilation may still be required to reduce hair scattering effects but the dark melanin-containing hair follicles are no longer present. However, this additional breeding takes time and additional cost, and it is not the optimal breeding plan for some transgenic mice strains.

In this work, we quantitatively examined the attenuation of bioluminescent signals due to skin pigmentation in C57B1/6 mice. Two studies were performed to systematically measure the levels of skin pigmentation in depilated transgenic $\mathrm{C} 57 \mathrm{Bl} / 6$ mice over time, and to determine how this affects the bioluminescent signal. The first study tracked the transmission of bioluminescent signal in a set of transgenic $\mathrm{C} 57 \mathrm{Bl} / 6$ mice through 5 weeks of the hair growth cycle, taking in vivo measurements every week. The second study used multiple groups of mice, each in a different stage of the hair growth cycle. In this latter study, bioluminescent signal transmission was measured ex vivo by placing the dorsal skin from sacrificed animals over a BLI simulating phantom. The phantom provided consistent and stable bioluminescent light, minimizing experimental variability.

We also explored a technique that can be used to compensate for signal loss in the skin. For this, reflectance spectroscopy was introduced, which is a simple method that is sensitive to light scattering and absorption changes in tissue, and was used here to estimate the level of melanin in the skin [16-21]. Measurements are made using a small, flexible fiber optic probe. One fiber in the probe delivers white light illumination to the surface of the tissue. Light then scatters through the sample, and a small fraction of the light is collected by a collection fiber, immediately adjacent to the illuminating fiber (Fig. 1). There are a wide variety of diagnostic applications for reflectance spectroscopy, often referred to as "elastic scattering spectroscopy," when the optical properties of the tissue are being investigated. This is generally distinguished from diffuse reflectance spectroscopy, where the source and detection fibers are separated by larger distances (typically $>5 \mathrm{~mm}$ ). In this geometry, signal is collected from deeper in the tissue, below the skin. For the purpose of this study though, we restricted our focus to melanin changes in the skin, to avoid signal fluctuations 
a)

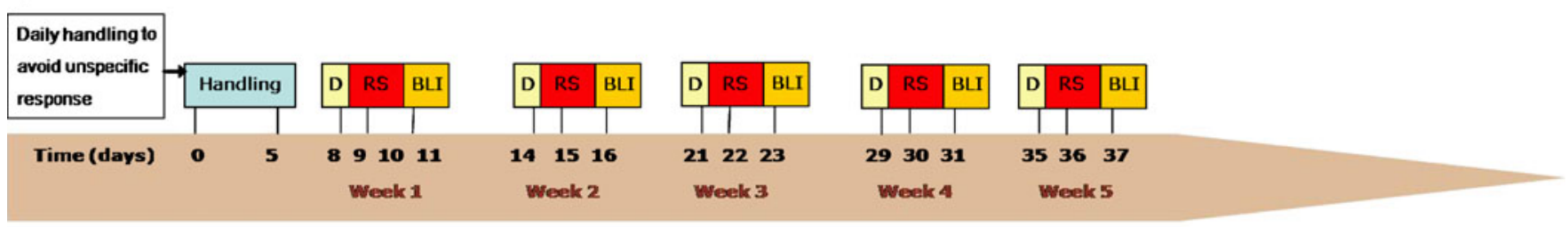

b)

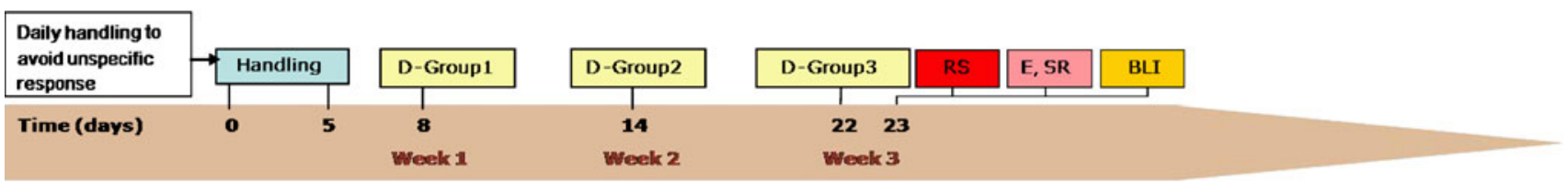

c)

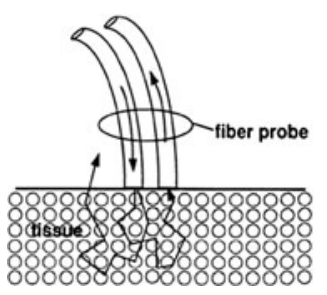

d)

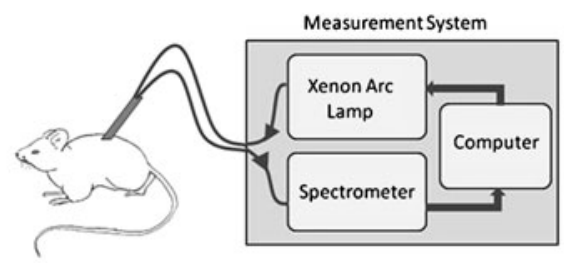

Fig. 1. a Illustration of in vivo study timeline. $D$ depilation, $R S$ reflectance spectroscopy measurements, $B L /$ bioluminescent imaging. b lllustration of phantom study timeline. $E$ euthanasia, $S R$ dorsal skin sample removal. c Schematic of the fiber probe used to acquire reflectance measurements. One fiber delivers broadband light, while the other collects the reflected light. $\mathbf{d}$ Schematic of the reflectance spectroscopy system, including a xenon broadband light source, and spectrometer.

from other non-melanin variations. The applications of reflectance spectroscopy range from in vivo detection of diseases like colon and pancreatic cancer, to in vitro monitoring of apoptosis in cell cultures [22-27]. Reflectance spectroscopy has also been used to investigate the optical characteristics of melanin in human skin [28, 29].

In the work presented here, the effects of skin pigmentation in $\mathrm{C} 57 \mathrm{Bl} / 6$ mice on accurately quantifying BLI signal loss is examined. In vivo reflectance measurements are used to estimate the amount of melanin in mouse skin. These reflectance measurements are collected in conjunction with BLI measurements, and the correlation between the reflectance signal and the percentage of BLI signal loss is examined. Ideally, this correlation function can be used to estimate the percentage of BLI signal loss, based on a reflectance measurement. Applying the predicted signal loss percentage to the measured BLI signal produces a corrected value that estimates the BLI signal in the absence of pigmentation. This procedure constitutes a simple correction method that can numerically compensate for signal attenuation. Use of such a method will allow for more flexibility and accuracy in bioluminescent imaging studies involving mice with pigmented skin by compensating for one source of measurement variability. This will reduce experimental error, which is particularly important during long-term studies and reducing number of animals per group. Overall, this paper highlights the significant effect that variations in mouse skin can have on a BLI study, and indicates that these variations should be taken into account in measurements, especially when sensitivity to small signal changes is required.

\section{Materials and Methods}

\section{Animals}

All animal handling and care was conducted in accordance with the Animal Care and Use Committee at the Novartis Institutes for BioMedical Research, Inc. in Cambridge, MA, USA. A total of 25 C57B1/6 transgenic mice were used for the studies, where luciferase expression is under the transcription control of a physiologically and pharmacologically inducible gene. All mice were housed under a normal $12 \mathrm{~h}$ light/dark cycle, and fed ad libitum standard rodent chow. Initial breeding mice were obtained from Charles River Laboratories (Wilmington, MA, USA), and the transgenic mice were created and bred at NIBRI for over six generations. Included in the in vivo study were four male and three female 2-month-old transgenic mice. The phantom study included nine male and nine female 5month-old transgenic mice. All mice were initially handled for a week prior to imaging to avoid any nonspecific response.

\section{Hair Removal}

The week following handling, mice were depilated. Mice were anesthetized with $2 \%$ isoflurane in oxygen, and all hair removal took place under a hood. A rectangular region of hair on the dorsal side, from the hind legs to the front legs, was first removed using an electric razor (Norelco G390, Philips), and the skin was then 
depilated using the chemical Nair ${ }^{\circledR}$ (Church \& Dwight Co., Inc.). Occasional skin lesions were treated by veterinary care with triple antibiotic ointment (Taro Pharmaceuticals) and any lesions were avoided during reflectance spectroscopy measurements.

\section{Histology}

At 2-weeks post-depilation, nine transgenic C57Bl/6 mice (four males, five females) were euthanized by cervical dislocation while under deep anesthesia (isoflurane). The dorsal skin (from the hind to front legs) was sampled, pinned to a corkboard, and fixed in $4 \%$ paraformaldehyde. Skin samples were then routinely processed to paraffin block, sectioned at $4 \mu \mathrm{m}$ and stained with hematoxylin and eosin (H\&E) for light microscopy examination.

\section{Bioluminescent Imaging}

In vivo Study All bioluminescent data was acquired with the Xenogen IVIS200 (Caliper Life Sciences, Hopkinton, MA, USA) imaging system. Five $\mathrm{mg}$ of $\mathrm{K}^{+}$Salt D-Luciferin (Caliper Life Sciences, Hopkinton, MA, USA) was dissolved in $5 \mathrm{ml}$ DPBS (Invitrogen), and filtered through a $0.2 \mu \mathrm{m}$ sterile filter (Millipore). Five minutes prior to imaging, mice were injected intraperitoneally (equally distributed left and right) with $8 \mathrm{mg} / \mathrm{kg}$ of the luciferin solution. Animals were then placed in an anesthesia induction chamber with $2 \%$ isoflurane in oxygen flowing at $150 \mathrm{ml} / \mathrm{min}$ for 5 min, timed with a stopwatch, and then transferred to the IVIS200 system and placed dorsally, while connected to nose cones. Photographic images were acquired immediately prior to bioluminescent imaging, to allow for anatomical localization of the signal. Image acquisition times were kept constant as bioluminescent signal was measured with the cooled CCD camera for 2 min. Following the imaging procedures, animals were placed in a separate cage and allowed to recover before being returned to their own cage. Two BLI images were taken during week 1 to account for any increase in baseline signal caused by possible unspecific response from handling. This imaging procedure was repeated over 5 weeks to assess the temporal effects of changes in skin pigmentation on the BLI signal (Fig. 1a). Week 1 and consecutive weeks refers to data taken the day after original hair removal.

Phantom Study In this study, 18 mice were divided into three groups (three males and three females per group), each being depilated during a different week of the hair cycle (Fig. 1b). A total of 3 weeks of depilation were included in this study to capture the transition from telogen to anagen phase. The day after the last group was depilated, reflectance spectroscopy measurements were taken in vivo on all mice. Animals were then euthanized by cervical dislocation under deep anesthesia. Dorsal skin samples were removed and each was placed over the center of the bioluminescence-mimicking light source, which was located on the lower left dorsal side of the BLI phantom (Caliper Life Sciences). BLI images were taken with a 1-s exposure time. This procedure was repeated for all three groups of animals. To ensure consistency, histological examination was performed to confirm that the excised skin samples were uniform in layer composition. Weeks 1 , 2, and 3 data refers to data that was collected from groups depilated for the first time on the day before the study, 1 week prior to the study, and 2 weeks prior to the study, respectively.

\section{Grayscale Values}

Grayscale values were recorded as a quantitative measure of our visual interpretation of skin pigmentation. Using Living Image 2.50.02 software (Caliper Life Sciences), regions of interest were drawn over the depilated region of photographic images and the average grayscale value, as defined by average counts per pixel, was used as a measure of skin pigmentation level. Image acquisition time was kept constant at $0.2 \mathrm{~s}$. Pigmentation was found to be relatively consistent over the entire region of interest, so an averaged grayscale measurement was deemed appropriate.

\section{Reflectance Spectroscopy}

Fig. 1d is a schematic of the reflectance spectroscopy system used in this study. It is composed of a pulsed xenon arc lamp (Perkin Elmer LS1130-3) for the light source, and a spectrometer with a linear CCD detector (Ocean Optics S2000). These are controlled by an on-board computer with display screen. A fiber probe is used for delivery and collection of light; it comprises two optical fibers with core diameters of $200 \mu \mathrm{m}$, numerical aperture values of 0.22 , with a center-to-center separation of $250 \mu \mathrm{m}$. The tip of the probe is faceted at $45^{\circ}$ which, when in optical contact with the skin, limits the photon penetration depth to approximately $300 \mu \mathrm{m}$. This allows for measurements that are sensitive primarily to the layers of skin while minimizing effects from underlying tissues [18]. To account for system response, a reference measurement is taken on a solid material that diffusely reflects equally at all wavelengths (Spectralon, Labsphere). The collection of data involves sending a burst of light through the illumination fiber, and the photons undergo a few scattering events in the tissue before being collected by the collection fiber. The collected light is measured between 400 and $700 \mathrm{~nm}$, and is defined as the reflectance spectrum of the sample. In general, increased scattering in a sample results in increased reflectance, and increased absorption results in decreased reflectance. The collected reflectance spectrum relates to a combination of both factors. We expect that an increase in melanin will result in decreased reflectance.

All reflectance measurements were taken while the mice were under anesthesia ( $2 \%$ isoflurane in oxygen), and before acquiring the photographic and BLI images, in both studies. The mice were probed at two separate locations on their dorsal side. These were consistently taken over the left shoulder blade and just above the right hip.

\section{Data Analysis}

Image acquisition and BLI data analysis were performed using the Living Image 2.50.02 Software (Caliper Life Sciences, Hopkinton, MA, USA). Additional computational analysis was done in Microsoft Office Excel 2007, and GraphPad Prism 4 (Graphpad Software, Inc., La Jolla, CA, USA). All reflectance spectroscopy data were acquired using internally developed software, and analyzed using Matlab software (Mathworks, Natick, MA, USA). The measured spectra were converted to relative reflectance values by normalizing to the reference measurement from Spectralon, which accounts for system response, using a method described earlier (see ref. [18]). This ensures that the measurements are independent of the amount of incident light. For the BLI images, a rectangular region of interest was defined over the entire dorsal skin area, and the average radiance was taken as the measurement. One-way ANOVA with Bonferroni post-tests were performed to compare measure- 
ments from week-to-week. Statistical significance is based on $p$ values, with $p \leq 0.05$ considered a significant difference.

Grayscale values are reported as the average number of counts per pixel collected in the photographic region of interest, which correlates to the depilated area. Reflectance values are given as the relative reflectance intensity at $560 \mathrm{~nm}$, the peak emission wavelength of firefly luciferase. BLI measurements are reported as the average

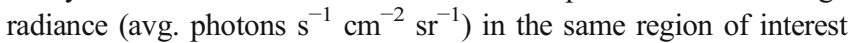
used for grayscale measurements. To enable grayscale and reflectance measurements to be compared to changes in BLI signal, BLI values are normalized to the baseline (i.e., week 1) BLI measurements for each animal respectively, and are given as a percentage.

Correlation between reflectance measurements and BLI signal loss was modeled as a linear function with the form:

$$
\mathrm{BLI}_{\% \text { Loss }}=A *(\text { Reflectance } \text { Measured })+B .
$$

The corrected BLI signal was calculated as:

$$
\mathrm{BLI}_{\text {Predicted }}=\mathrm{BLI}_{\text {Measured }} /\left(\mathrm{BLI}_{\% \text { Loss }} / 100\right)
$$

These equations constitute a simple correction method. With experimentally pre-determined values of A and B (ideally provided from the literature), Eqs. 1 and 2 can be combined to estimate BLI intensity in the absence of melanin. Percent error results were calculated between predicted BLI values and the average BLI signal of all mice in the phantom study week 1 group.

\section{Results}

\section{Histology}

Microscopic examination of the skin of a subset of transgenic mice showed that depilation induces the anagen phase of the hair growth cycle. The presence of melanocytes and melanin pigments in bulbs of fully developed hair follicles in the anagen phase accounted for the dark skin pigmentation noted macroscopically (Fig. 2). This is consistent with other findings in the literature [30].

\section{In vivo Imaging Study}

To monitor bioluminescence signal attenuation due to skin pigmentation over time, BLI, reflectance and grayscale data were collected from a group of seven mice (four female, three male) on a weekly basis for 5 weeks. The photographic images illustrate the evident increase in pigmentation from week 1 to 3 , returning again to baseline levels by week 5 (Fig. 3b). This trend is also demonstrated in the reflectance spectra plots, with the lowest reflectance values reported during week 3 , returning to week 1 levels in weeks 4 and 5 (Fig. 3a). This data illustrates that the pigmentation of mouse skin due to depilation is very consistent and predictable over a 5-week hair cycle.

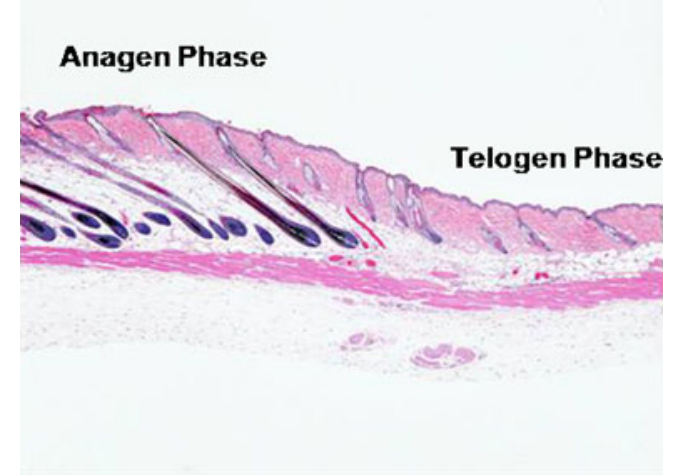

Fig. 2. H\&E staining of male $\mathrm{C} 57 \mathrm{BI} / 6$ dorsal skin sample at 2 weeks post-depilation. The right half shows telogen phase, and the left half shows anagen phase. The assumed source of skin pigmentation is from the collection of melanin in the hair follicles during the anagen phase.

Since we were not delivering any exogenous stimuli to induce the gene in our transgenic mice, we expect the BLI signals to have similar, low intensities across all weeks. The temporal trends between grayscale and reflectance values were similar, showing high signals for weeks 1, 2, 4, and 5, and significantly lower signals during week 3 , with a threefold, and fourfold decrease in signal, respectively (Fig. 4). For both grayscale and reflectance values, statistically significant differences were found between weeks 1 and $3(p<0.001, p<0.01$, respectively $)$. This agrees with visual observation of the photographic images and reflectance spectra in Fig. 3. Data from GS and reflectance revealed good correlation $\left(R^{2}=0.8\right)$, confirming that reflectance measurements are a good quantitative representation for our visual observation of pigmentation (Fig. 4).

In contrast, however, the BLI signal temporal trend is notably different from those of GS and reflectance measurements. Weeks 2, 3, 4, and 5 are all significantly different from week $1 \quad(p<0.001)$, with a six-, five-, three-, and twofold signal difference, respectively. Unlike the grayscale and reflectance trends, the BLI signal only reaches as high as $41 \%$ of initial baseline levels by week 5 . This discrepancy illustrates the significant experimental error that results from variability of BLI signal generation from week to week in our in vivo model. Therefore, these results are not representative of the actual signal attenuation, and thus correlation between BLI measurements and reflectance measurements are not informative.

\section{Phantom Study}

To provide a more systematic determination of light attenuation, a mouse-shaped tissue-equivalent phantom was used as the bioluminescence light source in the phantom study as a way to control baseline signal variation. In this study, three groups of mice, each representing a different week of the hair growth cycle, and each consisting of three male and three female mice, were used for a total of 18 


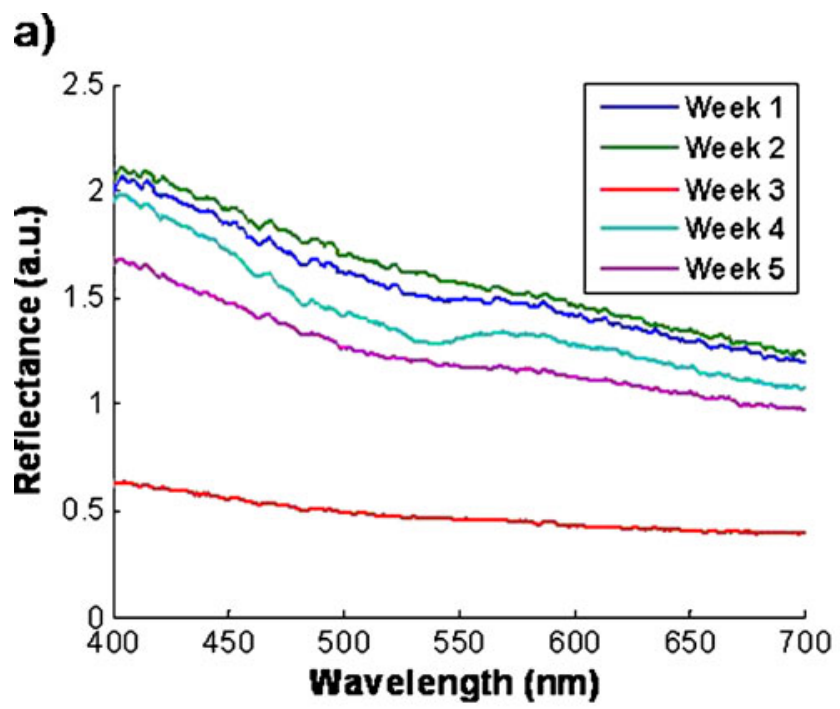

b)

Week 1

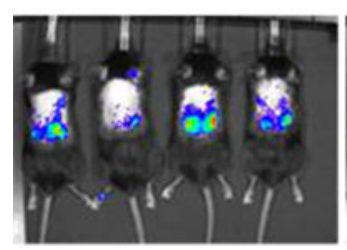

Week 2

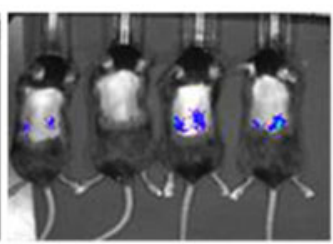

Week 3

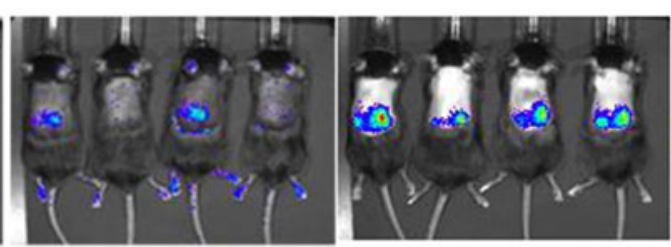

Week 4

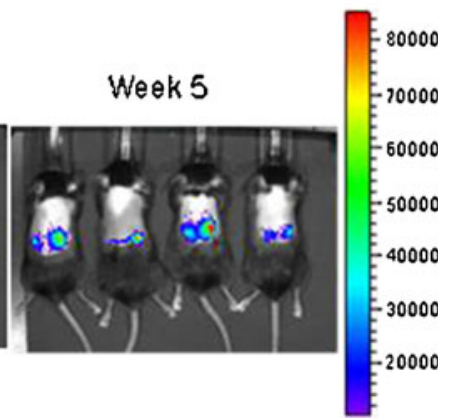

Fig. 3. a Sample of relative reflectance spectra over 5-week period from a male mouse in the in vivo study. b Sample photographic and BLI images for all female mice in the in vivo study. Photographic images show an increase in pigmentation at week 3 , and a full recovery to week 1 pigmentation levels by week 5 .

animals. A representative set of BLI images from each group is shown in Fig. 5, and Fig. 6a presents the temporal signal trends for BLI, grayscale, and reflectance signals. Grayscale and reflectance measurements are presented in the same manner as for those in the in vivo study. BLI measurements are also reported as in the in vivo study, but because the same mice were not studied over multiple weeks, the BLI signals were normalized to the average BLI signal from all mice in the week 1 group.

Comparable to the in vivo results, grayscale and reflectance measurements show minimal change between weeks 1 and $2( \pm 4 \%)$, and a statistically significant reduction in signal during week $3(p<0.001)$, which corresponds to high skin pigmentation (Fig. 6a). The BLI signal trend also shows low signal during week 3 and higher signals in weeks 1 and 2. BLI signal differences between all weeks are statistically significant $(p<0.001)$. Note in Fig. 5 that, at week 3, signal attenuation is so great that the skin shows no visible BLI signal transmission due to the limits of the false color scale dynamic range of the image. From this we find that, on average, strongly pigmented skin, such as that measured in the week 3 group, causes a 90\% attenuation of BLI signal, evidently an amount that can cause significant experimental errors.

Correlation between the measurements was examined for the purpose of finding a correction factor. As in the in vivo study, grayscale and reflectance measurements were well correlated $\left(R^{2}=0.62\right)$. By eliminating experimental variation in the BLI source, correlations of reflectance versus BLI, and grayscale versus BLI, were improved from those in the in vivo study (Fig. 6b). The correlation between reflectance values and BLI attenuation was found to be slightly stronger than that between grayscale and BLI signals. This may be partially attributed to temporal intensity fluctuations of the light source for grayscale measurements. Reflectance measurements take these variations into account by normalizing the spectra with a signal response measurement from the calibration phantom. Therefore reflectance spectroscopy is the more reliable source of melanin estimation.

Using the relationship between reflectance and BLI signal loss, corrected BLI values were calculated using Eq. 2, but with a $40 \%$ average error between the corrected and expected BLI values. Errors were magnified in cases of small reflectance values and large percent attenuation 

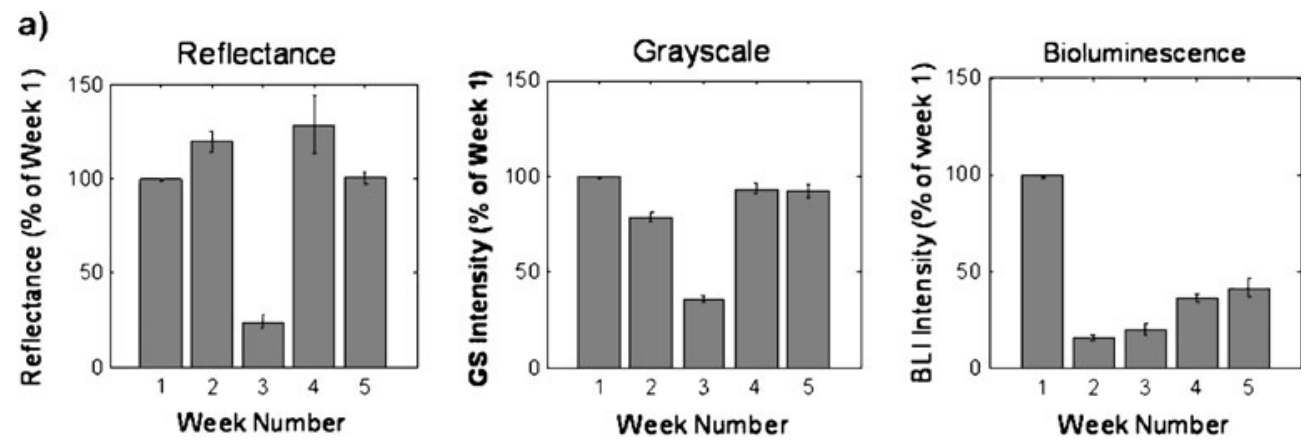

b)

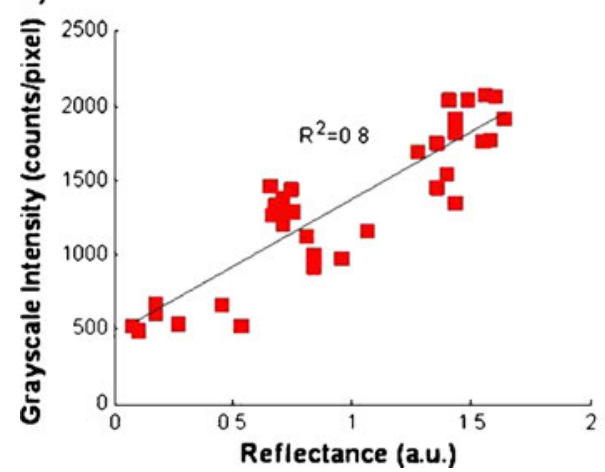

Fig. 4. a Temporal signal changes for BLI, reflectance spectroscopy, and grayscale measurements. Signal values averaged over all mice during each week and plotted along with SEM. Reflectance and grayscale measurement trends are similar, while the BLI signal trend is significantly different. b Correlation between grayscale and reflectance values indicates that reflectance measurements are a good representation of our visual interpretation of pigmentation levels.

predictions. Thus, these correlation equations are not strong enough to use in a reliable correction method.

\section{Gender Differences in Skin Response to Depilation}

To better understand the cause of the high variability, the role that gender played in these studies was further investigated. The data from the phantom study was separated by gender and plotted to examine correlation (Fig. 7). While BLI values were comparable between males and females, reflectance measurements were found to be 1.5 times larger in males as compared to females. This resulted in data that

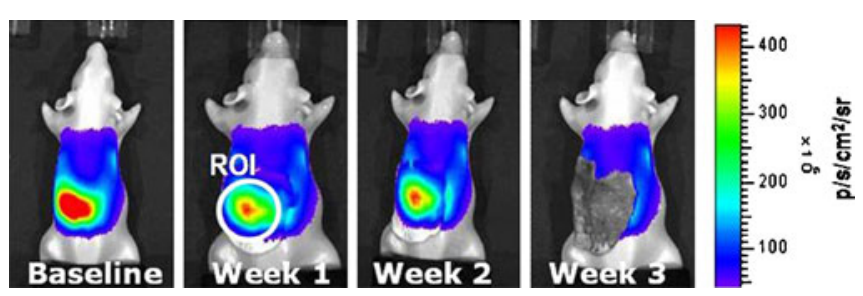

Fig. 5. Phantom photographic images with BLI signal overlaid. Skin sample is placed over light source in week 1 , week 2, and week 3 images. BLI measurements taken as the average signal from the ROI marked. The lack of BLI signal through the highly pigmented skin sample in week 3 illustrates the significant attenuation effects of melanin. was well separable, and correlation between reflectance values and BLI attenuation was greatly improved for each gender. $R^{2}$ correlation values rose from 0.66 with no gender separation to 0.73 and 0.92 in this analysis, for females and males, respectively. Similar results were observed in the grayscale vs. BLI correlations with gender separation, but with slightly poorer correlation values. Corrected BLI values still had errors of $33 \%$ and $39 \%$ for females and males, respectively.

\section{Full Spectral Analysis of Reflectance Measurements}

In the previous analysis, reflectance data was reported at a single wavelength $(560 \mathrm{~nm})$, ignoring the data collected from all other wavelengths. However, this broadband data is useful in characterizing the general composition of the skin. Comparison of broadband spectra can help identify differences in optical and physiological properties between tissues, and is used here to further explore the differences between pigmented and non pigmented skin. For each mouse in the in vivo study, the spectra from weeks 2, 3, 4, and 5 were normalized by the original spectrum from week 1 . The average of all normalized spectra for all males is presented in Fig. 7b. The plots representing weeks 2, 4, and 5, are clustered around unity, and have limited shape changes, indicating that the scattering and absorption 

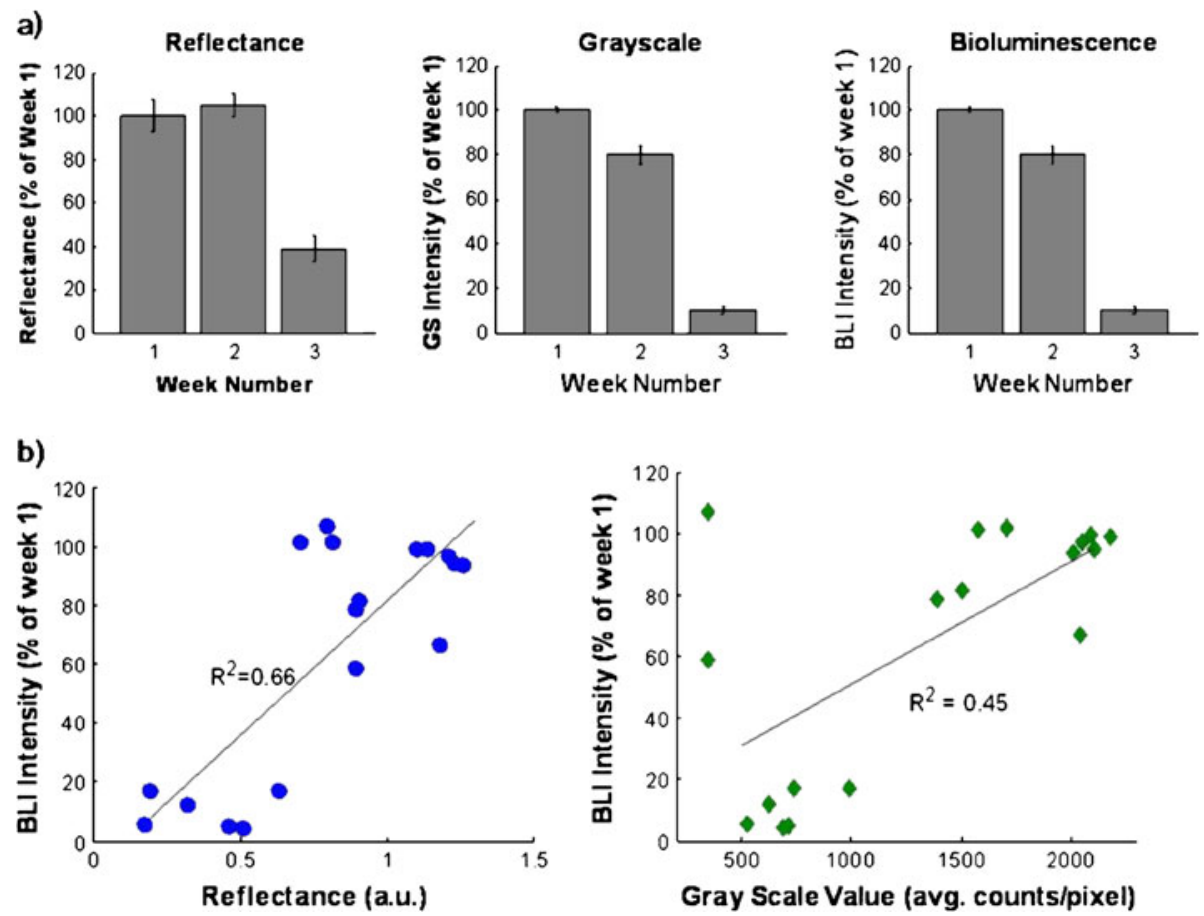

Fig. 6. a Temporal variations in signal from the phantom study. Signal values averaged over all samples during each week and plotted along with SEM. All three measurement modalities share a similar temporal trend. b Correlation of BLI versus reflectance, and BLI versus grayscale measurements.

properties of mice in these weeks are relatively similar to those in week 1 . The plot of week 3 is much lower, which indicates a higher degree of absorption, consistent with the presence of melanin in the skin when the measurement was taken. It also exhibits a slight positive slope.

\section{Discussion}

In the present study, the effects of melanin pigmentation in mouse skin on bioluminescence signal attenuation of transgenic C57B1/6 mice were examined during two studies that tracked skin through different stages of hair growth. Pigmentation was quantified using grayscale analysis of photographic images, and reflectance spectroscopy measurements. Both measurements were compared to bioluminescence signal loss. Initially, the in vivo study tracked a group of mice over 5 weeks of their hair growth cycle. This study illustrated that the pigmentation of mouse skin due to depilation is consistent and predictable over the 5 -week hair growth cycle. However, for the purpose of determining a possible correction factor, an accurate measurement of BLI attenuation due to melanin is not possible using our in vivo model alone, because of its inherent biological variability. To minimize variability, a stable bioluminescence phantom was substituted as the signal source, thus reducing an intrinsic source of experimental error. Using this in the
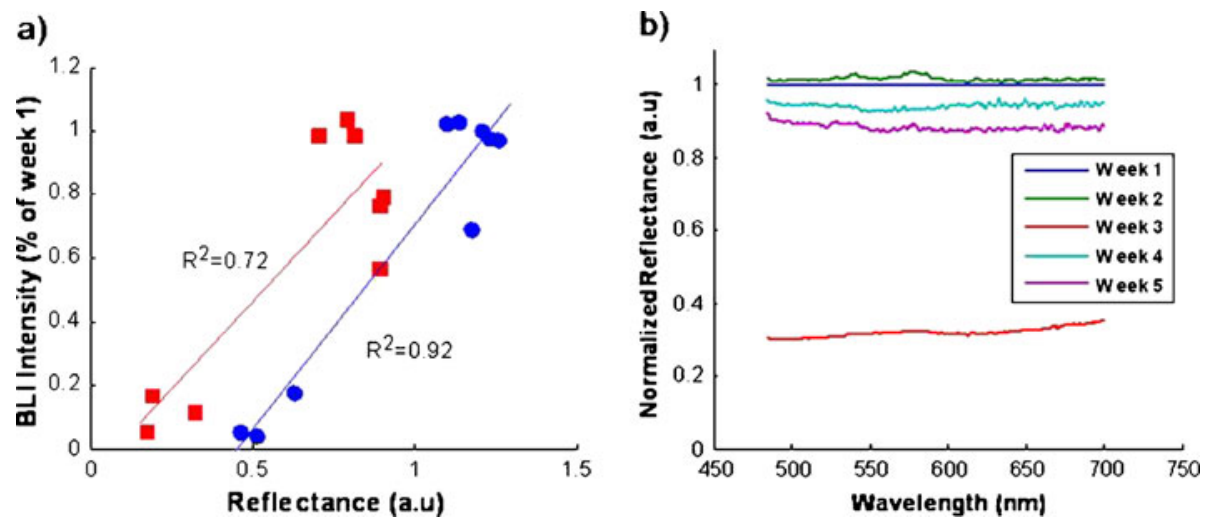

Fig. 7. a BLI and reflectance signal correlation, separated by gender, suggesting some experimental variability is the result of gender differences (squares, females; circles, males). b Reflectance spectra from all weeks, normalized by week 1; average of all males. Normalized plots highlight changes in tissue properties from week 1. 
phantom study, groups of mice, each representing a different stage in the hair growth cycle, were examined. Results revealed that heavily pigmented skin contributes to an average $90 \%$ loss in bioluminescent signal as a result of melanin absorption. Unfortunately, correlation between reflectance measurements and bioluminescence attenuation was not strong enough to form a reliable correction factor. A separation of data based on gender improved correlation. However, using these correlations to correct for BLI signal attenuation still resulted in significant error.

The observed poor correlation between reflectance measurements and BLI signal loss can be attributed to several factors. First is the fundamental difference in measurement geometry between BLI (transmission geometry), and gray scale/reflectance spectroscopy (reflection geometry). The tissue-photon optical interaction of BLI signal originating far below the skin surface is significantly different from that of photons collected in the reflection geometry, where signal is confined to the skin. Thus, the collected signal will represent different scattering and absorption properties. Ideally, a technique to quantify melanin levels for use in a correction method should collect signal in the transmission geometry to best emulate the BLI signal path. Transmission measurements, however, are not practical for in vivo applications due to the difficulty in positioning the skin between the illumination and collection fibers.

Another significant factor to consider is that of variation in tissue scattering properties. According to Hansen et al. [30], during transition from the anagen phase to the telogen phase, not only is there an increase in melanin content, but the combined skin thickness of the mouse also increases by approximately $63 \%$. The dermal layer, which is composed of high levels of collagen, accounts for over half of this increase. Collagen is a highly scattering medium, which produces high reflectance values. The decrease in reflectance due to melanin absorption in the anagen phase is thus partially offset by the increased amount of highly scattering collagen. This effect may also explain the slightly higher reflectance measurements reported in weeks 2 and 4, as illustrated in Fig. 4. During these transition weeks (from telogen to anagen, and anagen to telogen, respectively), both the melanin concentration and dermal thickness are changing. Depending on the relative rates of change, the increased scattering from collagen may offset the absorption from melanin, resulting in higher reflectance signals as compared to weeks 1 and 5. Increased scattering may also be attributed to the inherent scattering properties of melanin. Melanin's high refractive index, coupled with its packing in hair follicles, produces significant Rayleigh scattering [31].

Increased scattering from thicker skin layers also accounts for the differences in reflectance measurements between males and females. As has been reported, male mice have a dermal skin layer that is $50 \%$ thicker than that of females, and this difference results in a significant increase in scattering, and thus reflectance, due to a higher amount of collagen $[32,33]$. Such gender differences not only explain the source of some experimental variability, but they also reveal the importance of controlling for gender in experimental design by using a single gender, or by analyzing data from males and females separately.

Variation in non-melanin absorption also contributes to signal inconsistencies, and can be attributed to changes in blood volume/oxygenation, and in the concentration of compounds like bilirubin and beta carotene (to a much lesser extent). Some of this absorption variability can be seen in Figs. 3a and 7b where the shape of the weeks 4 and 2 spectra, respectively, show small shape inconsistencies. However, variations in non-melanin absorption appear much less significant than variations in scattering.

The combined effects of these scattering and absorption variations can be observed with full spectral analysis of the reflectance data (Fig. 7b), particularly in the plot representing week 3 (normalized to week 1). The lower amplitude of the plot reflects the significant melanin absorption, while the slight positive slope is due to the decreasing absorption coefficient of melanin with wavelength; at shorter wavelengths, melanin absorbs more strongly than at longer wavelengths, resulting in less reflectance at shorter wavelengths [11]. Due to the strength of attenuation, however, one would expect a steeper slope. We hypothesize that the shallower slope is the result of increased scattering signal. Like melanin absorption, scattering is also stronger at shorter wavelengths, counteracting the absorptive effects of melanin. In these studies, the combination of increased scattering and absorption neutralizes any dramatic shape change.

The observations reported here demonstrate that the effects of scattering and absorption are highly intertwined with one another, and that variability from non-melanin sources limits the reliability of raw reflectance measurements as an estimate of melanin concentration. To achieve stronger correlation between reflectance measurements and BLI attenuation, it is necessary to isolate the melanin contribution by separating the effects of scattering and absorption. Fortunately, a more complex reflectance model has recently been developed that is capable of separating scattering and absorption contributions through examination of the broadband reflectance signal [18]. This model is capable of estimating the relative concentration of melanin, which can then be used in our proposed correction method (Eqs. 1 and 2) in place of the raw reflectance value. It is promising that this approach will improve correlation and provide a reliable method to compensate for BLI signal loss. One of the challenges in implementing this method is in determining the extinction coefficient spectrum of mouse melanin, since the spectrum is needed as an input to the model (along with the extinction coefficient spectra of all other absorbers). Initial findings suggest that the spectral shape is quite different in $\mathrm{C} 57 \mathrm{Bl} / 6$ mice as compared to humans. This is a reasonable observation based on the visual difference in the color of human melanin (brown) as compared to melanin in our mouse model (gray). We are currently investigating this for a future paper. Because 
broadband analysis is not possible with grayscale measurements, a correction method using digital grayscale photographs is not likely. In additional future work, we plan to examine strain-specific differences in skin response due to depilation, comparing C57Bl $/ 6$, white-haired CD-1 mice, and nude mice. These results will allow us to determine the exclusive roles that melanin and hair follicles play in light attenuation through the skin.

\section{Conclusion}

Systematic quantitative analysis of bioluminescence absorption through C57Bl/6 mouse skin illustrates that signal can be attenuated up to $90 \%$ in heavily pigmented skin, as compared to lightly pigmented skin. Variability in the degree of signal loss due to melanin content will introduce significant error, especially in long-term studies. Assessment of melanin content in vivo is achieved using reflectancebased optical methods. When controlling for BLI source generation, temporal trends in bioluminescence signal loss and reflectance measurements agree with each other and with expectations based on visual observation. A correction method based on the correlation of bioluminescence signal loss and reflectance signal is proposed to estimate signal loss, and to numerically compensate for attenuation. However, we illustrate that advanced broadband analysis of reflectance measurements is necessary to make such a quantitative method reliable. We conclude that skin pigmentation effects in in vivo bioluminescent imaging studies are significant and should be taken into consideration when designing and implementing longitudinal studies, particularly if sensitivity to small signal changes is important.

Conflict of Interest. The authors declare that they have no conflict of interest.

Open Access. This article is distributed under the terms of the Creative Commons Attribution Noncommercial License which permits any noncommercial use, distribution, and reproduction in any medium, provided the original author(s) and source are credited.

\section{References}

1. Sato A, Klaunber B, Tolwani R (2004) In vivo bioluminescence imaging. Comp Med 54:631-634

2. Sadiko RT, Blackwell TS (2008) Bioluminescence imaging modality for in vitro and in vivo gene expression. Methods Mol Biol 477:383394

3. Klerk CPW, Overmeer RM, Niers TMH et al (2007) Validity of bioluminescence measurements for noninvasive in vivo imaging of tumor load in small animals. Biotechniques 43:7-13

4. Paroo Z, Bollinger RA, Braasch DA et al (2004) Validating bioluminescence imaging as a high-throughput, quantitative modality for assessing tumor burden. Mol Imaging 3:117-124

5. Rehemtulla A, Stegman LD, Cardozo SJ et al (2000) Rapid and quantitative assessment of cancer treatment response using in vivo bioluminescence imaging. Neoplasia 2:491-495

6. Contag C, Ross BD (2002) It's not just about anatomy: in vivo bioluminescence imaging as an eyepiece into biology. J Magn Reson Imaging 16:378-387

7. Contag $\mathrm{CH}$, Bachmann $\mathrm{MH}$ (2002) Advances in in vivo bioluminescence imaging of gene expression. Annu Rev Biomed Eng 4:235-268
8. Luker GD, Beib DA (2005) Luciferase real-time bioluminescence imaging for the study of viral pathogenesis. Methods Mol Biol 292:285-294

9. Inoue Y, Izawa K, Tojo A et al (2006) Light emission requires exposure to the atmosphere in ex vivo bioluminescence imaging. Mol Imaging 5:53-56

10. Alonso L, Fuchs E (2006) The hair cycle. J Cell Sci 119:391-393

11. Kollias N, Baquer A (1985) Spectroscopic characteristics of human melanin in vivo. J Invest Dermatol 85:38-42

12. Colin M, Moritz S, Schneider H et al (2000) Heamoglobin interferes with the ex vivo luciferase luminescence assay: consequence for detection of luciferase reporter gene expression in vivo. Gene Ther 7:1333-1336

13. Ntziachristos V, Bremer C, Weisslader R (2003) Fluorescence imaging with near-infrared light: new technological advances that enable in vivo molecular imaging. Eur Radiol 13:195-208

14. Jansen ED, Pickett PM, Mackanos MA et al (2006) Effect of optical tissue clearing on spatial resolution and sensitivity of bioluminescence imaging. J Biomed Opt 11:041119

15. Kato M, Takeda K, Kawamoto Y et al (2001) RET tyrosine kinase enhances hair growth in association with promotion of melanogensis. Oncogene 20:7536-7541

16. Bigio IJ, Mourant J (1997) Ultraviolet and visible spectroscopies for tissue diagnostics: fluorescence spectroscopy and elastic scattering spectroscopy. Phys Med Biol 42:803-814

17. Taroni P, Pifferi A, Torricelli A et al (2003) In vivo absorption and scattering spectroscopy of biological tissues. Roy Soc Ch 2:124-129

18. Reif R, A'Amar O, Bigio IJ (2007) Analytical model of light reflectance for extraction of optical properties in small volumes of turbid media. Appl Opt 46:7317-7328

19. Zonios G, Dimou A (2006) Modeling diffuse reflectance from semiinfinite turbid media: application to the study of skin optical properties. Opt Express 14:8661-8674

20. Mourant JR, Fuselier T, Boyer J et al (1997) Predictions and measurements of scattering and absorption over broad wavelength ranges in tissue phantoms. Appl Opt 36:949-957

21. Sun J, Fu K, Wang A et al (2006) Influence of fiber optic probe geometry on the applicability of inverse models and experimental measurements. Appl Opt 45:8152-8162

22. Mulvey CS, Curtis AL, Singh SK et al (2007) Elastic scattering spectroscopy as a diagnostic tool for apoptosis in cell cultures. IEEE J Sel Top Quantum 13:1663-1671

23. Dhar A, Johnson K, Novelli $M$ et al (2005) Elastic scattering spectroscopy for the diagnosis of colonic lesions: initial results of a novel optical biopsy technique. Gastrointest Endosc 63:257-261

24. Wilson R, Chandra M, Scheiman J et al (2009) Optical spectroscopy detects histological hallmarks of pancreatic cancer. Opt Express 7 (20):17502-17516

25. Arifler D, MacAulay C, Follen M et al (2006) Spatially resolved reflectance spectroscopy for diagnosis of cervical precancer: Monte Carlo modeling and comparison to clinical measurements. J Biomed Opt 11:064027

26. Arifler D, Schwarz R, Chang S et al (2005) Reflectance spectroscopy for diagnosis of epithelial precancer: model-based analysis of fiber-optic probe designs to resolve spectral information from epithelium and stroma. Appl Opt 44:4291-4305

27. Canpolat M, Akyuz M, Gokhan GA et al (2009) Intra-operative brain tumor detection using elastic light single-scattering spectroscopy: a feasibility study. J Biomed Opt 14:054021

28. Zonios G, Dimou A (2008) Melanin optical properties provide evidence for chemical and structural disorder in vivo. Opt Express 16:8263-8268

29. Zonios G, Dimou A, Bassukas I et al (2008) Melanin absorption spectroscopy: new method for noninvasive skin investigation and melanoma detection. J Biomed Opt 13:014017

30. Hansen LS, Coggle JE, Wells J et al (1984) The influence of the hair cycle on the thickness of mouse skin. Anat Rec 210:569-573

31. Wolbarsht ML, Walsh AW, George G (1981) Melanin, a unique biological absorber. Appl Opt 20:2184-2186

32. Azzi L, El-Alfy M, Martel C et al (2005) Gender differences in mouse skin morphology and specific effects of sex steroids and dehydroepiandrosterone. J Invest Dermatol 124:22-27

33. Calabro KW, Curtis A, Galarneau J et al (2010) Gender variations in the optical properties of skin in murine animal models. J Biomed Opt (in press) 